Cambouis, la revue des sciences sociales aux mains sales

\title{
Pratiques et politiques de la négociation pour accéder et se maintenir sur un terrain d'enquête
}

\section{Agnès Aubry}

Membre du Centre de Recherche sur l'Action Politique de l'Université de Lausanne (CRAPUL), chercheuse invitée FNS au Graduate Center, City University of New York (CUNY)

agnes.aubry@unil.ch

\section{Morgane Kuehni}

Professeure à la Haute école de travail social et de la santé de Lausanne HES.SO | HETSL

morgane.kuehni@hetsl.ch

\section{Laure Scalambrin}

Collaboratrice scientifique HES et boursière post-doctorat du domaine Travail Social de la HES.SO, HES.SO | HETSL

laure.scalambrin@hetsl.ch

Date de publication : $8 / 7 / 2021$

Dossier: Pratiques et politiques de la négociation pour accéder et se maintenir sur un terrain d'enquête

Comment citer : $10.52983 /$ crev.vi0.71

Licence:Cambouis publie ses contenus selon les termes de la Licence Creative Commons Attribution - Pas d'Utilisation Commerciale - Pas de Modification 4.0 International. Les auteurices gardent leurs droits de propriété intellectuelle pleine et entière sur leurs articles. 


\section{Pratiques et politiques de la négociation pour accéder et se maintenir sur un terrain d'enquête}

Agnès Aubry

Membre du Centre de Recherche sur l'Action Politique de l'Université de Lausanne (CRAPUL), chercheuse invitée FNS au Graduate Center, City University of New York (CUNY)

agnes.aubry@unil.ch

\section{Morgane Kuehni}

Professeure à la Haute école de travail social et de la santé de Lausanne HES.SO | HETSL

morgane.kuehni@hetsl.ch

\section{Laure Scalambrin}

Collaboratrice scientifique HES et boursière post-doctorat du domaine Travail Social de la HES.SO, HES.SO | HETSL

laure.scalambrin@hetsl.ch

Ce dossier de la revue Cambouis porte sur la question de la négociation d'un terrain d'enquête ethnographique. Mobilisée dès les années 1960 en sociologie de part et d'autre de l'Atlantique, ce type d'enquête, qui s'inscrit dans une longue tradition anthropologique, suppose une immersion des chercheur.euses sur leur terrain pour y récolter des données sur un temps relativement long. La négociation de l'accès et du maintien sur un terrain ethnographique s'est alors révélée être une thématique cruciale en sciences sociales, et peut être qualifiée de « classique », à double titre.

D'une part, il est aujourd'hui largement admis que la négociation d'entrée est une étape charnière dans la construction d'une recherche, car elle conditionne la possibilité même de l'enquête (Derbez, 2010). Les manuels d'enquête qualitative et les articles à visées méthodologiques s'accordent sur l'importance du point d'entrée, sur la nécessité de réfléchir aux modes de prise de contact, aux choix des interlocuteurs et interlocutrices et aux contraintes de « présentation de soi » (Goffman, 1973) et de son projet pour pouvoir accéder et se maintenir sur un terrain d'enquête (voir par ex. Grawitz, 1993 ; Beaud et Weber, 1997 ; Arborio et Fournier, 1999 ; Olivier de Sardan, 2008 ; Peneff, 2009 ; Dereze, 2009 ; Weber, 2009). L'enjeu n'est pas seulement de se faire admettre et accepter auprès des personnes ou du groupe que l'on souhaite étudier, mais aussi d'être « conscient du côté où l'on se trouve » lorsque l'on négocie un terrain d'enquête, comme l'écrit Henri Peretz (2004, p. 48) qui rappelle le danger de certaines « alliances initiales ».

D'autre part, davantage qu'un point de vigilance, cette thématique peut aussi être qualifiée de classique dans la mesure où elle renvoie aux compétences d'analyses et aux compétences réflexives des chercheur.euses. Muriel Darmon écrit par exemple que les négociations sont certes un préalable nécessaire, mais qu'« elles peuvent aussi être construites comme un objet de plein droit de la recherche et devenir ainsi un véritable matériau d'analyse du terrain lui-même ». D'illustres travaux ont montré que l'objet de recherche n'est pas dissociable des conditions d'enquête (Bizeul, 1998 ; Schwartz, 2012 
[1990]) et que « la signification des signes est le produit d'un contexte et d'une interaction », comme l'affirme Nicolas Jounin (2014, p. 73). Si les guides d'enquête de terrain encouragent les chercheur.euses à prêter une attention particulière aux arguments qui convainquent ou qui crispent leurs interlocuteur.trices, aux réactions suscitées par leur présence, mais aussi à leurs propres réactions, ce n'est pas seulement parce que les échanges préalables conditionnent l'enquête, mais aussi parce qu'ils constituent une première définition instructive de la situation d'enquête, façonnant les résultats ultérieurs (Favret-Saada, 1977 ; Peneff, 2009). Si l'analyse des réactions qu'engendre la présence de l'ethnographe est utile à la compréhension des mondes sociaux observés, plusieurs auteur.es se sont interrogé.es sur les modalités et les enjeux de cette présence, en particulier pour révéler ce que Georges Devereux nommait « les perturbations dans l'enquête » (Devreux, 1980). Plus récemment, des chercheur.euses ont finement relaté comment ces « épreuves ethnographiques » (Fassin, 2008) constituent une manière de mieux comprendre et d'analyser les faits sociaux (Bensa et Fassin, 2008 ; Bennani-Chraïbi, 2010). Les formes de l'« engagement ethnographique » (Cefaï, 2010) ont largement été travaillées en sociologie politique, qu'il s'agisse de l'engagement militant de celles et ceux qui s'adonnent à l'observation participante d'une mobilisation collective (Broqua, 2009 ; Massicard, 2002 ; Péchu, 2006 ; Havard-Duclos, 2007), de l'implication dans un parti politique (Aït-Aoudia et al., 2010) ou, plus largement, de l'engagement scientifique dans « l'arène sociale » (Fassin, 1999). Questionnant la pluralité « des liens politiques, institutionnels, professionnels, contractuels, mais aussi biographiques, familiaux, intimes et parfois même charnels qui peuvent intervenir dans la construction d'une recherche sociologique et dans sa réalisation » (Naudier et Simonet, 2011, p. 5), la notion d'engagement renvoie également à la position nécessairement située et incarnée des chercheur.euses (Villani et al., 2014). La démarche ethnographique implique des contraintes et des opportunités différenciées selon l'âge, le sexe, le statut académique ou l'expérience, par exemple (Monjaret et Pugeault, 2014; Hanson et Patricia, 2019). Ces réflexions ont largement irrigué la littérature ces dernières années, encourageant les chercheur.euses à re-questionner les productions académiques en mettant en lumière les contextes dans lesquels les objets sont choisis et les savoirs sont fabriqués (Haraway, 1988 ; Collins, 2000 ; Ahmed, 2006). Que l'enquête ethnographique s'inscrive dans des terrains réputés « sensibles » (Bouillon, Fresia et Tallio, 2005), « difficiles » ou « faciles » (Boumaza et Campana, 2007), qu'elle soit confrontée à d'éventuelles craintes ou attentes des personnes enquêtées, les négociations façonnent les relations d'enquête et les connaissances qui en résultent. Si l'ensemble de ces aspects ont pu être traités dans le domaine des sciences humaines et sociales, pourquoi consacrer aujourd'hui un numéro thématique à la question de la négociation d'un terrain d'enquête?

Le premier apport de ce numéro peut être qualifié de «pratique », intégrant une visée à la fois méthodologique et pédagogique. Les processus de négociation pour accéder et se maintenir sur un terrain de recherche sont aujourd'hui encore souvent passés sous silence dans les rapports de recherche et les publications. Ouvrir la «boîte noire » de la fabrication des enquêtes pour rendre visibles les pratiques de négociation nous semble d'autant plus important qu'elles peuvent être très anxiogènes compte tenu de leur caractère déterminant. En témoignent les incertitudes persistantes de nombreux·euses chercheur.euses lorsqu'il s'agit d'entamer une négociation de terrain : comment s'y prendre ? Vers qui se tourner? Quelles sont les conséquences directes et indirectes des stratégies mobilisées sur les interactions, sur le dispositif d'enquête et les données recueillies ? Il est aujourd'hui largement admis que « l'enquête ethnographique reste, la plupart du temps, une aventure solitaire et, semble-t-il, impossible à standardiser » (Beaud et Weber, 2012, p. 231) et 
qu'il « est impossible d'appliquer des "recettes a priori” » (Peretz, 2004, p. 49). Est-il d'ailleurs seulement possible pour les chercheur.euses d'expliciter tout ce qui a été fait pour accéder et se maintenir sur un terrain d'enquête?

Dans ce numéro, nous avons pris cette question au sérieux en réunissant des contributions se focalisant sur les démarches de négociation, explorant ses formes et ses atours à différents moments de l'enquête. Il ne s'agit pas de recenser les « bonnes pratiques », mais de donner à voir et à comprendre comment les chercheur.euses s'y sont pris.es pour accéder et se maintenir sur leur terrain d'enquête. Cette démarche s'inscrit dans la lignée des analyses réflexives entreprises ces dernières années sur la « réhabilitation » des difficultés méthodologiques (Guionnet et Rétif, 2015), autour des obstacles (Chamboredon et al., 1994), des « incidents » (Bouillon, Fresia et Tallio, 2005), des « ratés de terrain » (Stavo-Debauge, Roca i Escoda et Hummel, 2017) ou encore des «malentendus » (Papinot, 2007) provoqués par la négociation et la présence des chercheur. euses sur leur terrain d'enquête. Nous prêtons alors non seulement attention aux échecs et aux difficultés, mais nous questionnons également ce qui fonctionne de prime abord sans encombre.

Donnant à voir et à comprendre la négociation comme une relation sociale et comme un processus itératif et continu (entrée, maintien, sortie du terrain d'enquête), il s'agit de mettre en avant les formes de « compromis relationnels réciproques » (Guillaume et Pochic, 2011, p. 121) qui permettent aux chercheur.euses de pouvoir accéder à un terrain (parfois inespéré), et de faire durer leur présence ${ }^{1}$. Dans ce sens, nous utilisons le terme de «négociation » pour désigner les processus de communications, de transactions et d'échanges entre au moins deux parties (un.e chercheur.euse et son terrain) dont l'objet concerne une entente ou un accord relatif à l'établissement et aux modalités d'une relation d'enquête. Cette démarche procède d'une action intentionnelle de la part des chercheur.euses pour rendre leur présence possible. Ils et elles peuvent solliciter une autorisation formelle ou un accord informel, exposer leurs questions de recherche et leurs méthodes d'enquête ou rester discrets et discrètes, estimant que toute formalisation des raisons de leur présence nuirait à l'enquête : c'est notamment le cas des enquêtes « incognito » (Dargère, 2012 ; Le Lay, 2014) ou du «braconnage » (Jounin, 2014, p. 54). Les textes réunis dans ce numéro mobilisent le terme de négociation lorsque les échanges avec les terrains sont explicites. Ils mettent en exergue les contextes dans lesquels ces échanges prennent place, soulignant les modalités toujours co-construites des processus de négociation, et leur dépendance à un cadre qui dépasse les acteurs et actrices en présence (Rolle, 2016). Négocier est un verbe d'action ; cette action sous-tend le pouvoir d'agir des individus, mais celle-ci s'inscrit toujours dans un jeu de positions et de relations de pouvoir qui structurent cette relation (Bourque et Thuderoz, 2011).

Le second apport de ce numéro peut être qualifié de «politique». Notre ambition n'est pas seulement de revenir sur la position nécessairement située et incarnée des chercheur.euses et sur leurs plus ou moins grands succès dans la négociation d'entrée et de maintien sur leur terrain, mais aussi d'inscrire les pratiques de négociations dans un débat plus large. Mettre en mots les pratiques de négociation permet de faire émerger une série de questionnements et de positionnements parfois transversaux aux différents terrains d'enquête et d'interroger les différents enjeux que recouvre la démarche ethnographique dans un champ académique en mutation. Les discussions et les éventuelles prises de position concernant les pratiques de négociation peuvent être détachées des cas particuliers et rapportées à une série d'évolutions qui font peser certaines exigences sur la recherche dans ce domaine. De nombreux travaux ont par exemple souligné des évolutions majeures dans le champ des sciences
1 Sur les négociations invisibles, voir par ex. Olivier de Sardan, 1995. 
sociales : apparition de comités d'éthique, contractualisation des rapports de recherche, judiciarisation des conflits (Gagnon et Eysermann, 2012 ; Laurens et Neyrat, 2010 ; Perrin et al., 2018 ; Roca i Escoda et al., 2020).

Outre les questions de faisabilité et de formalisation, voire de pression à la standardisation du travail de recherche, ces évolutions marquent fortement le travail des ethnographes sur le terrain et en questionnent la nature. Alors que nous nous devons par exemple de donner les gages du caractère éthique de nos recherches, pouvons-nous seulement promettre notre «inoffensivité » pour les institutions, comme pour les enquêté.es? Dans un contexte de fortes injonctions à la transparence, se pose la question de la possibilité même de réaliser une immersion entièrement transparente : « les ethnographes doiventils en dire plus à leur enquêtés que les gens ordinaires, dans les situations ordinaires, qui comportent toujours une part d'opacité ?» (Chauvin, 2017).

La négociation dépasse toujours le cadre de la relation entre un·e chercheur.euse et un terrain d'enquête et renvoie également à des enjeux qui convoquent la sphère dite de l'« intime ». Cette négociation de soi à soi est par exemple très perceptible quand les chercheur.euses doivent abandonner un terrain (Rolle, 2017) ou opérer des arbitrages dans des situations délicates ou lorsqu'ils et elles sont dans des situations de « dilemmes éthiques » (Bouquet, 2003). Donnant à voir ce qui est bien souvent relégué au domaine du « privé », et passé sous silence dans les productions scientifiques, nous proposons de discuter explicitement de la manière dont les sociologues font face à des préoccupations professionnelles, matérielles, affectives et personnelles (Günel, Varma et Watanabe, 2020). Cette option peut être qualifiée de politique dans la mesure où elle vise à décloisonner les frontières du dicible et de l'indicible, de l'oralité et de l'écriture (Clair, 2016), du personnel et du professionnel. Partant du principe que les affects et les émotions qui irriguent et façonnent les différentes phases de la négociation ne sont pas des « encoubles » au travail ethnographique mais en sont une condition, nous souhaitons aborder « l'inconfort sociologique» (Bourrier, 2010, p. 31), les tourments et les hésitations, ou encore, les sentiments de culpabilité vis-à-vis des enquêté.es.

\section{Organisation du dossier}

Ce dossier est né de la rencontre entre les éditeurs de cette nouvelle revue qui promeut explicitement de mettre au jour les pratiques de recherche (y compris les plus « sales » au sens de « sale boulot» d'E. Hughes (1996)) et les organisateurs et organisatrices de la troisième édition des Ateliers lausannois d'ethnographie (ALE) ${ }^{2}$ en mai 2018 à Lausanne, dont l'objectif était d'explorer la question de la négociation de l'accès aux terrains de recherche en sciences sociales. Selon la ligne éditoriale de la revue, ce numéro est ouvert à d'autres contributions, qui peuvent proposer d'approfondir certains des enjeux mentionnés plus haut, notamment. Il réunit à l'heure actuelle sept textes et deux entretiens, et se décline en quatre parties.

\section{Négociations et (dés) engagements}

Les deux contributions réunies dans ce premier axe reviennent sur les liens unissant pratiques de la négociation et engagements dans des milieux d'interconnaissance plus ou moins formalisés : le «monde de la rue » d'une ville de l'Est de la France (Besozzi) et une section locale du Parti Socialiste français (Delasalle). Partant du postulat que l'engagement à long terme dans le monde quotidien et routinier des autres est une condition méthodologique sine qua non de l'ethnographie (Emerson, 1987, p. 71), cet axe donne à voir comment la nature et les modalités pratiques de cet engagement varient d'un terrain et
2 Créés en 2015, les ALE sont un lieu de réflexion sur les modalités de l'enquête ethnographique et sur ses enjeux théoriques, pratiques et éthiques. Le format « atelier » a été choisi afin de privilégier des communications courtes, suivies d'échanges avec les discutant·es et le public. Les interventions des participant.es mobilisent des matériaux de «première main », issus d'un travail de terrain avancé ou au contraire terminé. Ces matériaux doivent permettre d'articuler des réflexions de nature méthodologique ou épistémologique avec des données situées. In fine, les matériaux peuvent se présenter sous différents aspects : séquences ethnographiques, compterendu d'une situation d'enquête, extrait(s) d'entretien, séquence vidéo, bande-son, photographie(s), etc. Les échanges autour de la question de la négociation d'un terrain d'enquête ont bénéficié du soutien financier du Fonds national suisse de la recherche scientifique (FNS). 
d'un.e ethnographe à l'autre. Les contributions de Kevin Delasalle et Thibaut Besozzi soulignent toutes deux les négociations et ajustements constants que suppose l'engagement ethnographique (Cefaï, 2010), que ceux-ci s'effectuent avec soi-même ou avec les enquêté.es.

Les articles mettent tout d'abord en lumière la manière dont les modalités de l'engagement des chercheurs évoluent durant les différentes phases de l'enquête. Les deux auteurs montrent que le degré de leur implication sur le terrain varie, selon qu'il s'agit de négocier son entrée, son maintien ou sa sortie. Les deux contributions mettent également en avant des façons différenciées de s'engager dans le monde des autres. De quelle manière s'investir afin d'être accepté dans un monde duquel on est a priori éloigné ? Faut-il choisir le camp dans lequel on s'engage ? L'engagement devient-il nécessairement plus conséquent à mesure que l'enquête avance? De quelles manières souligner son implication envers les enquêtées, comment donner des gages de sa sincérité ? Les articles de cet axe permettent d'entrevoir la multiplicité des formes que peut prendre l'engagement ethnographique, selon les nécessités du terrain et les transactions dans lesquelles les sociologues se trouvent pris·es. Les arbitrages menés par chacun des chercheurs, à mesure que certains dilemmes se posent, les amènent à suivre des voies différentes. D'une façon originale, la question de la négociation de la sortie du terrain est notamment traitée ici à l'aune du désengagement - affectif, militant - que cela suppose.

Si les deux auteurs répondent à ces questions de façon circonstanciée, ils rappellent, en filigrane, que les modalités de l'engagement sont tributaires, d'une part, du rapport qu'entretiennent les ethnographes à leur terrain d'enquête et, d'autre part, de leur disponibilité biographique. Les « coûts » et les « risques » de l'engagement ethnographique ont en effet toutes les chances de varier selon que les chercheur-euses ont « un emploi à plein-temps, un mariage ou une famille » (McAdam, 1986, p. 70), notamment. Dans ce cadre, les « qualités » des sociologues (Naudier et Simonet, 2011) sont importantes à relever, ce que ne manquent pas de faire les deux contributions que l'on se propose ici de mettre en perspective.

Enquêtant sur « le monde de la rue » de la ville de Nancy, milieu duquel il est socialement éloigné, Thibaut Besozzi a redoublé d'efforts afin de réussir les premières interactions tenues avec ses enquêtés (majoritairement des hommes). Ces « premières impressions », qui selon Goffman (1973) cadrent le reste des interactions, sont importantes tant elles conditionnent les possibilités d'accès et de maintien sur le terrain et, partant, la récolte de données. Pour Thibaut Besozzi, la négociation de l'accès au terrain passe par un travail conscient de sa présentation de soi. Il décide d'adapter son style vestimentaire, sans pour autant se déguiser en SDF ni dissimuler son identité de chercheur ; le rôle investi pour négocier sa présence doit en effet être « acceptable » pour les enquêté.es (Hughes, 1996, p. 267). Tout en ne pouvant effacer totalement la distance qui le sépare des personnes qu'il est amené à rencontrer, il s'engage dans les relations sociales en adoptant ce que Brunetaux nomme « des postures de communes humanités » (Brunetaux, 1995). Au fur et à mesure, l'auteur adapte «sa posture, l'expression du visage, les gestes qu'il effectue » (Nizet et Rigaux, 2014, p. 45), ce qui a l'avantage de lui permettre « d'apprendre par corps » (Faure, 2000), en étant parfois « traité comme un sans-abri » par différent.es acteur.trices, extérieur.es au milieu d'interconnaissance dans lequel il est engagé.

L'auteur mesure néanmoins son engagement auprès des un.es et des autres afin d'éviter les situations d' « enclicage », conduisant à être assimilé à une « clique » ou une « faction » du milieu étudié (Olivier de Sardan, 1995). Ce faisant, il maintient et souligne une posture d'extériorité, ce qui lui est d'autant moins dommageable qu'il s'approche de la fin de son terrain. Il (se) rappelle ponctuellement qu'il est « là » pour sept-huit mois, durée du man- 
dat de recherche qui lui a été attribué. Cette présence limitée dans le temps facilitera par ailleurs la négociation de sa sortie ou son désengagement du milieu étudié.

Kevin Delasalle fait le pari inverse pour se maintenir sur son terrain partisan : une section du Parti Socialiste (PS) français. Dans son cas, l'enclicage est, dit-il, « devenu progressivement inéluctable dans la négociation implicite menée avec mes "enquêtés" pour me maintenir sur le terrain ». Afin de négocier sa présence sur le long terme, il doit alors «prendre parti » (Pudal, 1989)... ce qu'il fera, sans l'avoir anticipé, durant plus de six ans.

Du fait de sa socialisation familiale - son père a milité dans l'aile gauche du PS - mais aussi d'une recherche de Master 2 menée précédemment sur le rapport du PS aux classes populaires, Kevin Delasalle connaît le milieu qu'il souhaite étudier dans le cadre de sa thèse, ce qui en facilite l'entrée. Or, si les dispositions et affinités politiques des chercheur.euses façonnent le choix du terrain de manière plus ou moins implicite, celles-ci vont également conditionner les façons de mener l'enquête ainsi que « les modes d'observation participante » choisis (Dunezat, 2011). Les stratégies déployées par Kevin Delasalle pour se maintenir sur son terrain deviennent en effet de plus en plus marquées par le rapport militant qu'il entretient à son terrain politique : d'« ethnographe-militant », il devient petit à petit « militant-ethnographe ». En ce sens, l'engagement militant de l'ethnographe sur son terrain (Apoifis, 2016) constitue ici un moyen lui permettant d'y accéder et de s'y maintenir (Broqua, 2009), mais il est également une conséquence de son immersion.

Les différentes stratégies de négociation - plus ou moins conscientes - que Kevin Delasalle met en ouvre pour pouvoir mener une immersion de longue durée au sein d'une section du PS le mènent en effet à s'investir dans le travail militant du parti, notamment afin de « donner des gages de loyauté » à certain.es de ses enquêté.es. Au fur et à mesure, la prise en charge d'un nombre toujours plus important de tâches militantes fonctionne alors comme autant de paris adjacents (Becker, 2006) pour l'ethnographe : il est, petit à petit, projeté dans la carrière de collaborateur d'élu. Alors qu'il ne l'envisageait pas quelques mois ou quelques années plus tôt, il lui semble désormais difficile de retourner en arrière. Il tiendra son poste pendant trois ans. Ainsi « happé » par son terrain, la négociation de la sortie n'est alors possible qu'au prix d'un « désengagement militant» (Fillieule, 2005) qui est, entre-temps, devenu « coûteux ». Cela suppose comme il l'écrit de «[r] ompre définitivement avec un milieu militant qui avait occupé tant de place dans [sa] vie professionnelle et personnelle ». Sa défection sera finalement permise par l'existence de « possibles latéraux» (Lefebvre, 2008 ; Fillieule, 2005) : l'auteur doit écrire sa thèse et peut se réinvestir dans le milieu académique, et il fait l'expérience de déceptions militantes qui l'amènent à ressentir le même « tourment » que certain·es de ses enquêté·es.

\section{Les « fronts multiples » de la négociation}

Dans ce deuxième axe, les articles s'attachent à rendre compte des pratiques de négociation des chercheur.euses lorsqu'ils et elles s'adressent à des personnes ou des groupes qui n'occupent pas les mêmes positions dans le champ investigué. Teresa Brannick et David Coghlan (2007) parlent de « primary access » pour rendre compte de la permission formelle d'entrer dans une institution «fermée » (autorisation le plus souvent donnée par les personnes qui occupent des positions de pouvoir), et de « secondary access » pour définir l'accès aux personnes qui y travaillent et/ou à leurs publics. Plutôt que de partir d'une réflexion en termes de niveaux d'accès, nous proposons de questionner les «multi-fronts » de la négociation, mettant en exergue les pratiques des chercheur-euses travaillant à l'ouverture de plusieurs accès avec des enjeux 
différenciés, parfois contradictoires, et parfois simultanément. Dans cette perspective, la négociation n'est pas toujours linéaire, ni constituée d'étapes prédéfinies. Une ouverture d'un terrain d'enquête « au sommet » de la hiérarchie ne garantit pas forcément l'accès aux personnes occupant une position subordonnée dans l'espace social investigué, et inversement. Si les processus de négociation se renouvellent le plus souvent tout au long du terrain d'enquête, il s'agit dans cette deuxième section de prêter une attention particulière aux formes qu'elle peut prendre : quel est l'impact de la structuration interne des institutions, des niveaux hiérarchiques, des règles, des normes et des rapports de pouvoir sur les pratiques de recherche ethnographiques? Les stratégies de négociation reposent-elles sur l'adoption de stratégies de présentation de soi et de son travail spécifiques, différenciées selon le profil des enquêté.es, des chercheur.euses et de leur position dans les institutions? Comment les chercheur·euses ont-ils et elles procédé pour accéder à la parole d'enquêté·es sous « emprise institutionnelle»?

Les trois articles réunis dans cet axe proposent une «immersion » dans des terrains d'enquête multi-sites (Meyer, Perrot et Zinn, 2019) : les établissements scolaires (Rufin et Deshayes), la justice pénale des mineur.es (Weil, Bugnon et Frauenfelder) et les centres de santé sexuelle (Debergh). Ces trois recherches ont nécessité une négociation d'entrée formelle pour accéder aux enquêté.es, avec des enjeux fortement différenciés selon leur position dans la hiérarchie institutionnelle et socio-professionnelle, et selon les sites investigués. Grâce à une mise en perspective particulièrement riche entre les différentes institutions et au sein de celles-ci, les auteur.es font un effort d'explicitation remarquable des argumentaires mobilisés pour rendre la recherche possible auprès des directions, des professionnel-les et des usager-ères de différentes institutions. Leurs analyses réflexives de ces échanges mettent en avant les enjeux pratiques, épistémologiques, éthiques et déontologiques des pratiques de négociations.

À partir d'une recherche portant sur l'enseignement primaire genevois, l'article de Diane Rufin et Fabien Deshayes explore trois phases du processus de négociation : la conception du projet de recherche, l'entrée proprement dite sur le terrain d'enquête et la négociation interindividuelle avec les enseignant.es. S'intéressant aux effets de formalisation et de judiciarisation des processus de négociation sur la liberté et l'autonomie des chercheur.euses, les auteur.es montrent que la négociation directe avec la population enquêtée, construite dans le temps et sur la base d'une confiance réciproque, est bousculée par «l'inflation de dispositifs destinés à favoriser l'éthique de la recherche ». À ce titre, la crainte concernant l'accord du public - ici, les parents d'élèves - et leur nécessaire assentiment écrit, fait figure de révélateur, puisque certain·es professionnel.les s'érigent en « défenseurs des usagers ». Si ces réticences apparaissent avant tout comme une protection des institutions (Becker, 2016 [1983]), les auteur.es l'analysent également comme l'expression d'une forme de judiciarisation des relations sociales. Celle-ci influence très directement les pratiques de recherche, demandant notamment aux chercheur.euses de multiplier leurs efforts pour « accéder à une version non officielle de l'institution ». Dans cette enquête de longue durée, Rufin et Deshayes soulignent la progressive transformation du statut de l'enquête, passant d'une « source d'inquiétude » à une « source d'attentes » pour les enquêté.es.

Des processus relativement similaires sont mis en lumière par Armelle Weil, Géraldine Bugnon et Arnaud Frauenfelder dans leur enquête portant sur les « carrières pénales » des jeunes aux prises avec la Justice en Suisse romande. Dans leur article, les auteur.es s'intéressent à la « délicate posture des chercheur.euses lorsqu'ils.elles se trouvent entre plusieurs publics », les obligeant à « naviguer entre des fidélités parfois antagonistes ». Si les juges ont rapidement accepté leur projet d'enquête, la négociation fut en revanche 
plus compliquée avec les éducateur.trices, qui se sont érigé·es en « rempart de défense » des jeunes, décrit.es comme un public vulnérable. Dans une posture chère à l'ethnographie, les auteur.es montrent comment les difficultés d'accès aux enquêté-es permettent l'émergence de nouvelles pistes d'analyses. Armelle Weil, Géraldine Bugnon et Arnaud Frauenfelder mettent au jour un « certain mimétisme entre les sanctions pénales et la relation d'enquête »: dans les institutions fermées, davantage que dans les milieux ouverts, les chercheur.euses ont été soumis·es au respect des règles institutionnelles et à la « bonne volonté des professionnel-les » pour accéder aux jeunes. De leur côté, les jeunes s'intéressent moins au respect des protocoles d'enquête qu'aux «qualités morales » des enquêteurs.trices, montrant en «creux» les ressources fortement différenciées sur lesquelles peuvent s'appuyer les chercheur.euses. Comme le soulignent les auteur.es " gagner la confiance des enquêté.es dépend en effet moins d'un gage unique de "neutralité", d' "écoute", d' "empathie" ou de "bonne distance" que d'une multitude de paramètres et de mécanismes sociaux que les chercheur.es parviennent, de manière plus ou moins consciente et efficace, à identifier et à anticiper en situation d'entretien ».

Dans son article, Marlyse Debergh revient sur ses pratiques de négociation auprès de deux centres de santé sexuelle. Son article est particulièrement éclairant sur les « multi-fronts » de la négociation. Il montre les stratégies différenciées qu'elle a mobilisées auprès du premier centre, qui a pour publics cibles les hommes ayant des rapports sexuels avec d'autres hommes, et auprès du second qui est ouvert à tout public mais accueille essentiellement des jeunes femmes. Cet effort comparatif met en lumière un double contraste dans les processus de négociation : entre les deux centres selon leur degré d'institutionnalisation et de légitimité, d'une part, et, d'autre part, entre les négociations établies avec les directions et celles qui ont été menées avec les professionnel.les et les usager.ères. Ce second contraste met l'accent sur la dimension temporelle de la négociation et sa réversibilité : une négociation d'entrée aisée auprès de la direction ne détermine pas un accès facile aux publics et, inversement, une négociation d'entrée difficile, ne présage en rien des relations tendues par la suite. Au contraire dans l'enquête de Marlyse Debergh, tout se passe comme si la confiance gagnée de « haute lutte » ouvrait par la suite un accès facilité aux différents échelons de l'institution. Mobilisant un retour réflexif sur sa position située de chercheuse, elle met en lumière les effets fructueux de la proximité sociale et de genre sur les relations établies avec les usagères d'un des centres investigués. Elle en livre une très belle illustration à partir de l'accès aux corps des enquêté.es, montrant en quoi les enjeux différenciés de négociation sont révélateurs des rapports sociaux structurant plus largement les relations : si les corps des femmes lui sont plus spontanément dévoilés et ainsi rendus accessibles à l'observation, ce n'est pas le cas des corps des hommes, qui font moins volontiers l'objet d'un contrôle et d'une surveillance médicale.

\section{Les temps de la négociation}

Les deux contributions réunies dans le troisième axe reviennent sur les temps de la négociation, appréhendés ici comme le temps qui s'écoule inéluctablement (chronos) et le temps opportun, favorable à l'action (kairos). Les enquêtes restituées portent sur des «institutions fermées » qui nécessite une autorisation d'entrée sur le terrain, ce qui peut prendre du temps, ou ne jamais se réaliser. Travaillant respectivement sur l'Armée suisse et les délibérations du jury de concours de musique internationaux, Stéphanie Monay et Miriam Odoni reviennent en premier lieu sur la durée des négociations et les procédés concrets déployés pour négocier leur accès à des institutions a priori totalement fermées à l'observateur ou l'observatrice extérieure - le 
public, les civils - et au sein desquelles existe alors une véritable « culture de la restriction de l'information » (Deschaux-Beaume, 2011, p. 1), sinon du secret. En tentant d'accéder à ces terrains, les chercheuses mettent en jeu la hiérarchie institutionnelle et se confrontent à des univers officiellement régis par et constitués selon des asymétries de pouvoir. Leur présence ne peut alors se négocier sans une connaissance fine de ces asymétries et des enjeux qu'elles recouvrent, notamment afin de trouver les allié.es propices à l'ouverture du terrain car disposant d'une légitimité institutionnelle (Coton, 2018). Comment pénétrer, s'introduire et négocier son entrée dans des univers bureaucratiques, fortement hiérarchisés et particulièrement réticents à livrer des informations sur leur mode de fonctionnement? Quelles sont les adaptations voire les concessions nécessaires à la réalisation d'une enquête de terrain dans une institution dite « fermée » ? Les chercheuses montrent que le temps qui s'écoule (chronos) a également façonné les négociations dans le sens où il a eu un impact sur leur corps et leur statut.

Stéphanie Monay et Miriam Odoni montrent également que les institutions s'ouvrent aussi en fonction d'un certain nombre d'enjeux, propres aux temps de l'enquête. Dans ce sens, c'est kairos, le temps opportun, qui joue en faveur de l'ouverture du terrain. Les deux contributions soulignent de manière éclairante comment la visibilité croissante d'une critique, notamment médiatique, vis-à-vis du fonctionnement de ces institutions opaques et «muettes », peut constituer un véritable levier pour négocier son droit d'entrée. La levée du tabou concernant la culture institutionnelle sexiste des armées occidentales ou la critique du manque de transparence et d'ouverture des délibérations du jury de concours de musique internationaux, toutes deux relayées dans les médias, ont façonné les négociations respectives de Stéphanie Monay et de Miriam Odoni. Tout en bénéficiant d'un contexte d'ouverture de l'institution investiguée favorisant leur entrée, les auteures montrent comment elles se sont progressivement construites une « légitimité » et une « crédibilité » notamment scientifique, voire une « respectabilité » (Bruneteaux, 1995, p. 111) pour négocier leur présence dans des univers jusque-là fermés.

Enquêtant sur la socialisation sexuée des femmes militaires engagées volontairement dans l'Armée suisse, Stéphanie Monay doit éviter à tout prix de représenter « une menace pour l'image de l'institution militaire suisse ». Elle fait alors le choix (en partie contraint) de présenter sa recherche à l'une des gatekeepers de l'institution, « la femme la plus haute gradée de l'Armée suisse, la seule brigadière », comme étant « inoffensive ». En adoptant une posture ne pouvant être associée à celle « de l'intellectuel-le critique » (Pruvost, 2014), elle laisse ainsi penser que sa recherche peut véhiculer une image positive de l'institution, dans un contexte de visibilisation à la fois scientifique et militaire « des violences sexistes et sexuelles vécues par les militaires féminines dans le cadre des armées occidentales ».

De façon similaire, ce sont les critiques vis-à-vis de la trop grande opacité des délibérations des concours de musique internationaux qui vont ouvrir les portes à Miriam Odoni, après huit années de fermeture. Dans ce contexte, la chercheuse est mobilisée comme étant tout à la fois la preuve et la garante d'un processus de « démocratisation » de l'institution. Durant son enquête de terrain de longue haleine, face à des lieux clos - les salles des délibérations du jury - la chercheuse est contrainte tout d'abord « d'adopter une position de retrait et d'observatrice » silencieuse. Cette posture de « discrétion absolue » lui permet de développer une connaissance extrêmement fine du milieu enquêté, qui lui sera capitale au moment de rencontrer les membres du jury. Lorsque les portes s'ouvrent enfin, la chercheuse bénéficie d'un contexte singulier : un environnement « sous haute tension », où se manifeste la volonté de rendre plus transparents les concours de musique internationaux. À cela 
s'ajoutent des « enjeux internationaux de rationalisation et d'objectivation du jugement » auxquels son statut scientifique de sociologue (désormais diplômée d'un doctorat) semble entièrement répondre.

Les deux chercheuses soulignent, in fine, combien leurs propriétés sociales, et notamment leur sexe et leur âge (réel ou supposé), contraignent leurs pratiques de négociation dans des univers fortement et volontairement marqués par des hiérarchies et des rapports de pouvoir. Pour Stéphanie Monay, le fait de paraître «bien plus jeune que son âge »- une caractéristique a priori contraignante pour elle (Fournier, 2006) - lui a permis de renforcer son apparente « inoffensivité ». Mais la jeunesse apparente de l'enquêtrice l'a également contrainte à faire doublement montre de rigueur scientifique afin de garantir le sérieux de sa recherche et de sa «plus-value ». De son côté, Miriam Odoni souligne combien son accès aux délibérations d'un jury de piano est tributaire de sa métamorphose ou de son « évolution » au cours du temps. C'est bien le statut de docteure qui objective sa légitimité et son expertise auprès d'un jury constitué « de personnes de pouvoir aux carrières artistiques prestigieuses ». La construction d'une crédibilité scientifique pour ces chercheuses tient donc également une place importante dans les négociations leur permettant d'accéder et de se maintenir dans ces institutions.

\section{Entretiens avec Olivier Schwartz et Jean Peneff}

La quatrième section de ce numéro propose deux entretiens avec des chercheurs qui ont contribué à importer les méthodes ethnographiques en sociologie : Olivier Schwartz et Jean Peneff. Figures incontournables de la sociologie qualitative française, leurs récits permettent d'ancrer les pratiques de négociation à l'échelle d'un parcours de vie (professionnel et personnel), marqués par leurs longues expériences en tant que chercheurs et en tant qu'enseignants dans des universités françaises et à Alger (pour Jean Peneff). Se prêtant avec générosité au jeu de l'entretien, leurs témoignages mobilisent divers exemples relatifs aux différentes recherches qu'ils ont menées ou dirigées. Ils permettent d'inscrire leurs pratiques dans les milieux sociaux desquels ils sont issus et les contextes politiques et académiques dans lesquels ils ont évolué.

Les récits incarnés des deux auteurs répondent à l'ambition de ce numéro : dévoiler une part des « coulisses » de leurs pratiques de recherche, mais aussi les questionner sur les aspects plus politiques de la négociation et ce qui constitue à leurs yeux certains enjeux actuels des pratiques ethnographiques. Ces échanges offrent une mise en perspective particulièrement éclairante sur des questions épistémologiques ou déontologiques des pratiques de recherche, tout en soulignant les évolutions qu'a pu connaître la recherche dans le domaine des sciences sociales. La richesse de leur retour est incontestablement liée à leurs positions professionnelles actuelles : deux chercheurs officiellement à la retraite, dotés d'une forte légitimité dans le champ des sciences sociales. Les pratiques de négociation s'inscrivent incontestablement dans des cadres sociaux et professionnels qui sous-tendent la faisabilité du travail de terrain. Mais au-delà des enjeux propres au monde académique, et du constat d'une certaine précarisation des conditions de travail, les deux auteurs relèvent que les pratiques de recherche s'élaborent collectivement, que ce soit avec leurs collègues, ami-es ou conjoint.es. En ce sens, ce bilan est aussi l'occasion pour eux de souligner leur dette à l'égard de certaines personnes décisives dans leurs carrières.

Jean Peneff et Olivier Schwartz reviennent également sur l'importance d'être prêt.e à « mettre les mains dans le cambouis » pour se lancer dans une enquête ethnographique. Ils soulignent l'importance de devoir s'adapter aux temporalités et enjeux propres aux mondes étudiés. Sans tomber dans 
la survalorisation du côté valeureux d'une enquête de terrain (supposant souvent une vision androcentrée de la pratique ethnographique et de sa nécessaire prise de risque), ils soulignent la part inévitable d'inconfort dont les ethnographes font l'expérience lors des différentes phases d'une enquête, et ce, tout au long de leur carrière. Des capacités d'adaptation décrites par Jean Peneff lors des différentes phases de « mises à l'essai » par les professionnels hospitaliers, aux preuves et gestes d'attention d'Olivier Schwartz pour atténuer « ce que l'entretien, en face-à-face, pourrait avoir d'insécurisant » pour les conducteurs de bus de la RATP, les pratiques ethnographiques engagent fortement les chercheur.euses. Qu'il s'agisse de se sentir mal à l'aise en demandant à un·e enquêté·e de se prêter à l'exercice de l'entretien, ou d'être amené à, littéralement, se salir les mains afin de ne pas compromettre l'enquête, les moments entourant la négociation ne sont ainsi pas toujours confortables. De la même manière, mentir par omission ou faire le choix, en partie contraint, de mener l'enquête sous une fausse identité, soulève des questionnements qu'il n'est pas aisé de résoudre.

À rebours d'une tendance à l'extrême formalisation des pratiques, les deux chercheurs affirment qu'il n'existe pas une seule manière de répondre aux dilemmes éthiques qui apparaissent en cours de recherche. Ils plaident pour une sociologie à la fois curieuse et respectueuse des enquêté.es car l'« authentique souci » qui les anime constituent des boussoles permettant de prendre position sur certains enjeux propres à la démarche ethnographique. Si la science «implique des choix qui ne sont pas exclusivement de nature scientifique » (Fassin, 1999, p. 62), la façon dont nous faisons de la recherche dit quelque chose de la société dans laquelle nous voulons vivre (Kuehni, 2008). 


\section{Références}

Ahmed Sara, 2006, « Orientations : Toward a Queer Phenomenology », GLQ : A Journal of Lesbian and Gay Studies, vol. 12, nº4, p. 543-574.

AïT-Aoudia Myriam, BACHELot Carole, BARgel Lucie, Combes Hélène, Dechezelles Stéphanie, Ethuin Nathalie, Haegel Florence, LeClerco Catherine, Massicard Élise et Petitfils Anne-Sophie, 2010, « Enquêter dans les partis politiques. Perspectives comparées », Revue internationale de politique comparée, vol. 17, nº, p. 7-13.

ApoIf Is Nicholas, 2016, « Fieldwork in a furnace : anarchists, antiauthoritarians and militant ethnography », Qualitative Research, vol. 17, no 1, p. 3-19.

Arborio Anne-Marie et Fournier Pierre, 1999, L'enquête et ses méthodes : l'observation directe, Paris, Nathan, $128 \mathrm{p}$.

BEAUD Stéphane et WEBER Florence, 2012, «11 - Le raisonnement ethnographique » dans L'enquête sociologique, Paris, Presses Universitaires de France, p. 223-246.

BEAUD Stéphane et WEBER Florence, 1997, Guide de l'enquête de terrain, Paris, La Découverte, 288 p.

BECKER Howard, 2016 [1983], «Étudier l'école » dans Ethnographie de l'école. Les coulisses des institutions scolaires et scoio-éducatives, Rennes, Presses Universitaires de Rennes, p. 9-20.

BECKer Howard, 2006, « Notes sur le concept d'engagement », Tracés, [En ligne], $\mathrm{n}^{0} 11$.

BenNANI-ChraїBI Mounia, 2010, « Quand négocier l'ouverture du terrain c'est déjà enquêter. Obtenir la passation de questionnaires aux congressistes de partis marocains », Revue internationale de politique comparée, vol. 17, nº4, p. 93-108.

Bensa Alban et Fassin Didier, 2008, Les politiques de l'enquête, Paris, La Découverte (coll. « Recherches »), 336 p.

Bizeul Daniel, 1998, « Le récit des conditions d'enquête : exploiter l'information en connaissance de cause », Revue française de sociologie, p. 751-787.

BouIllon Florence, Fresia Marie et TALlio Virginie, 2005, Terrains sensibles. Expériences actuelles de l'anthropologie, Paris, CEA-EHESS.

Boumaza Magali et CAMPana Aurélie, 2007, « Enquêter en milieu "difficile". Introduction », Revue française de science politique, vol. 57, $\mathrm{n}^{\circ} 1$, p. 5-25.

BouQuet Brigitte, 2003, Éthique et travail social, Paris, Dunod, 230 p.

Bourque Reynald et Thuderoz Christian, 2011, Sociologie de la négociation. Nouvelle édition, avec études de cas, Rennes, Presses Universitaires de Rennes, 274 p.

BOURRIER Mathilde, 2010, « Pour une sociologie "embarquée" des univers à risque? », Revue de la société suisse d'Ethnologie, nำ15, p. 28-37.

Brannick Teresa et Coghlan David, 2007, «In Defense of Being "Native": The Case for Insider Academic Research », Organizational Research Methods, vol. 10, ${ }^{\circ} 1$, p. 59-74.

BRoQuA Christophe, 2009, «L'ethnographie comme engagement : enquêter en terrain militant», Genèses, vol. 75, n² 2, p. 109-124.

BRUNETEAUX Patrick, 1995, « Manœuvres scientifiques en terrain militaire », Genèses, n¹9, p. 108-121.

CEFAÏ Daniel, 2010, L'engagement ethnographique, Paris, Editions de l'EHESS. 
Chamboredon Hélène, Surdez Muriel, Pavis Fabienne et Willemez Laurent, 1994, « S'imposer aux imposants. A propos de quelques obstacles rencontrés par des sociologues débutants dans la pratique et l'usage de l'entretien », Genèses. Sciences sociales et histoire, p. 114132.

Chauvin Sébastien, 2017, « Les placards de l'ethnographe » dans En immersion. Approches ethnographiques en journalisme, littérature et sciences sociales, Rennes, Presses Universitaires de Rennes.

CLAIR Isabelle, 2016, « La sexualité dans la relation d'enquête. Décryptage d'un tabou méthodologique », Revue française de sociologie, vol. 57, $\mathrm{n}^{0} 1$, p. 45-70.

Collins Patricia Hill, 2000, Black Feminist Thought : Knowledge, Consciousness, and the Politics of Empowerment, New York, Routledge, $2^{\text {nd }}$ Edition.

CотоN Christel, 2018, « Classer la sociologue, déclasser les pairs. Pratiques et registres de distinction en terrain militaire », Genèses, vol. 110, nº 1 , p. 133-148.

DARGÈRE Christophe, 2012, L'observation incognito en sociologie. Notions théoriques, démarche réflexive, approche pratique et exemples concrets, Paris, L'Harmattan, $170 \mathrm{p}$.

Derbez Benjamin, 2010, « Négocier un terrain hospitalier. Un moment critique de la recherche en anthropologie médicale », Genèses, vol. 78, $\mathrm{n}^{0} 1, \mathrm{p} .105-120$.

DEREZE Gérard, 2009, Méthodes empiriques de recherche en communication, Bruxelles, De Boeck.

DeschauX-Beaume Delphine, 2011, « Enquêter en milieu militaire Stratégie qualitative et conduite d'entretiens dans le domaine de la défense », Res Militaris, vol. 1, n², http://www.resmilitaris.net/ ressources/10138/97/res_militaris_article_deschaux_texte_int_gral. pdf.

Devreux Georges, 1980, De l'angoisse à la méthode dans les sciences du comportement, Paris, Flammarion.

DunEzat Xavier, 2011, « Travail militant et/ou travail sociologique ? Faire de la sociologie des mouvements sociaux en militant» dans Des sociologues sans qualités ?, Paris, La Découverte, coll. « Recherches », p. 80-97.

EMERSON Robert M., 1987, « Four Ways to Improve the Craft of Fieldwork », Journal of Contemporary Ethnography, vol. 16, nº 1, p. 69-89.

FAssin Didier, 2008, « Introduction. L'inquiétude ethnographique » dans Les politiques de l'enquête, Paris, La Découverte, coll. « Recherches », p. 7-15.

FASSIN Didier, 1999, « L'anthropologie entre engagement et distanciation : essai de sociologie des recherches en sciences sociales sur le sida en Afrique » dans Becker Charles et al. (ed.), Vivre et penser le sida en Afrique, Paris-Dakar, Codesria/Karthala/IRD, p. 41-65.

FAURe Sylvia, 2000, Apprendre par corps. Socio-anthropologie des techniques de danse, Paris, La Dispute.

FAVRET-SAADA Jeanne, 1977, Les mots, la mort, les sorts. La sorcellerie dans le bocage, Paris, Gallimard.

Fillieule Olivier, 2005, Le désengagement militant, Paris, Belin.

FOURNIER Pierre, 2006, « Le sexe et l'âge de l'ethnographe : éclairants pour l'enquêté, contraignants pour l'enquêteur », ethnographiques.org, ${ }^{\circ} 11$, https://www.ethnographiques.org/2006/Fournier.

GAGNON Eric et EysERmanN Beatrice, 2012, « Trouver son intérêt, ou le perdre? », Éthique publique, vol. 14, $\mathrm{n}^{\circ} 1$, https://journals.openedition. org/ethiquepublique/936. 
Goffman Erving, 1973, La mise en scène de la vie quotidienne. 1. La présentation de soi, Paris, Éditions de Minuit.

Grawitz Madeleine, 1993, Méthodes des sciences sociales, 9? édition., Paris, Dalloz.

Guillaume Cécile et Pochic Sophie, 2011, « Peut-on enquêter sur l'égalité professionnelle sans intervenir? Retour sur une recherche en entreprise » dans Des sociologues sans qualités ?, Paris, La Découverte, coll. «Recherches », p. 117-133.

Guionnet Christine et RÉTIF Sophie, 2015, Exploiter les difficultés méthodologiques. Une ressource pour l'analyse en sciences sociales, Rennes, Presses Universitaires de Rennes, 176 p.

GÜNEL Gökçe, VARMA Saiba et Watanabe Chika, 2020, « A Manifesto for Patchwork Ethnography », Fieldsights, 2020, https://culanth.org/ fieldsights/a-manifesto-for-patchwork-ethnography.

Hanson Rebecca et Patricia Richards, 2019, Harassed. Gender, Bodies, and Ethnographic Research, Oakland, University of California Press, 240 p.

HarawaY Donna, 1988, « Situated Knowledges : The Science Question in Feminism and the Privilege of Partial Perspective », Feminist Studies, vol. 14, nº 3 , p. 575-599.

HAVARD-Duclos Bénédicte, 2007, « Les coûts subjectifs de l'enquête ethnographique. Enquêter comme militante dans l'association Droit Au Logement (DAL) à la fin des années 1990 », SociologieS, https:// journals.openedition.org/sociologies/182.

Hughes Everett, 1996, Le regard sociologique. Essais choisis, Paris, Éditions de l'EHESS.

Jounin Nicolas, 2014, Voyage de classes. Des étudiants de Seine-Saint-Denis enquêtent dans les beaux quartiers, Paris, La Découverte, 256 p.

KuEHNi Morgane, 2008, « Retour sur une exclusion de terrain d'enquête auprès de lingères : lorsqu'un terrain soulève la question du travail de la sociologue débutante, entre autre » dans Laboratoire du travail, Lausanne, Antipodes, p. 27-38.

LAURENS Sylvain et NEYRAT Frédéric, 2010, Enquêter : de quel droit? Menaces sur l'enquête en sciences sociales, Vulaines sur Seine, Éditions du Croquant, 320 p.

LE LAY Stéphane, 2014, « Enseignements empiriques et éthiques d'une biffe sociologique parmi les éboueurs parisiens », Actes de la recherche en sciences sociales, vol. 205, n ${ }^{\circ} 5$, p. 120-131.

LefebvRe Rémi, 2008, «Militer au Parti Socialiste pour le transformer. L'engagement à la "gauche" du PS » dans Laurent Willemez (ed.), Pour une gauche de gauche, Bellecombe-en-Bauges, Éditions du Croquant, p. 217-237.

MASSICARD Élise, 2002, « Être pris dans le mouvement : savoir et engagement sur le terrain », Cultures \& conflits, $\mathrm{n}^{\circ} 47$, p. 117-143.

McAdam Doug, 1986, « Recruitment to High-Risk Activism : The Case of Freedom Summer », American Journal of Sociology, vol. 92, nº 1, p. 6490.

Meyer Michaël, Perrot Adeline et Zinn Isabelle, 2019, « Entre ambition « tout-terrain » et impossible ubiquité : les ethnographes en mouvement. Introduction. », SociologieS, 2019, https://journals. openedition.org/sociologies/6521.

Monjaret Anne et Pugeault Catherine, 2014, Le sexe de l'enquête. Approches sociologiques et anthropologiques, Lyon, ENS Éditions, $300 \mathrm{p}$.

NAUDiER Delphine et Simonet Maud, 2011, Des sociologues sans qualités? Pratiques de recherche et engagements, Paris, La Découverte, coll. « Recherches », 256 p. 
Nizet Jean et Rigaux Natalie, 2014, La sociologie de Erving Goffman, Paris, La Découverte (coll. « Repères »), 2? éd., 128 p.

OlIVIER DE SARDAN Jean-Pierre, 2008, La rigueur du qualitatif. Les contraintes empiriques de l'interprétation socio-anthropologique, Louvain-La-Neuve, Academia-Bruylant, 368 p.

Olivier de SARdan Jean-Pierre, 1995, « La politique du terrain. Sur la production des données en anthropologie », Enquête, nºl, p. 71-109.

PAPINOT Christian, 2007, « Le "malentendu productif". Réflexion sur la photographie comme support d'entretien », Ethnologie française, vol. $37, \mathrm{n}^{\circ} 1$, p. $79-86$.

PÉchu Cécile, 2006, Droit Au Logement, genèse et sociologie d'une mobilisation, Paris, Dalloz.

Peneff Jean, 2009, Le goût de l'observation, Paris, La Découverte, coll. « Repères », 260 p.

Peretz Henri, 2004, Les méthodes en sociologie. L'observation, Paris, La Découverte, coll. « Repères », 128 p.

Perrin Julie, BüHLER Nolwenn, Berthod Marc-Antoine, Forney Jérémie, KRADOLFER Sabine et Ossipow Laurence, 2018, « Searching for ethics : legal requirements and empirical issues for anthropology », Tsantsa, nº23, https://frama.link/2nWBrUeZ.

Pruvost Geneviève, 2014, « Ni policier ni homme : une sociologue enquête sur la féminisation de la police » dans Le sexe de l'enquête : approches sociologiques et anthropologiques, Lyon, ENS Éditions.

PudAl Bernard, 1989, Prendre Parti. Pour une sociologie historique du PCF, Paris, Presses de la FNSP.

Roca I Escoda Marta, Burton-Jeangros Claudine, Diaz Pablo et Rossi Ilario, 2020, « Pour une éthique dans la recherche en sciences sociales » dans Enjeux éthiques dans l'enquête en sciences sociales, Genève, Université de Genève, coll. « Sociograph-Sociological Research Studies ».

Rolle Valérie, 2017, « Abandonner le terrain des bikers », SociologieS, https://journals.openedition.org/sociologies/6094.

RoLle Valérie, 2016, « Les corps professionnels en jeu. Quand le tatouage révèle les hiérarchies des univers enquêté et enquêteur », Genèses, vol. 104, n³, p. 115-132.

SCHWARTz Olivier, 2012, Le monde privé des ouvriers. Hommes et femmes du Nord, Paris, Presses Universitaires de France, 3? éd.

Stavo-Debauge Joan, Roca i Escoda Marta et Hummel Cornelia, 2017, «Enquêter. Rater. Enquêter encore. Rater encore. Rater mieux », SociologieS, https://journals.openedition.org/sociologies/6084.

Villani Michela, Poglia Mileti Francesca, Mellini Laura, Sulstarova Brikela et SINGY Pascal, 2014, « Les émotions au travail (scientifique): enjeux éthiques et stratégies méthodologiques d'une enquête en terrain intime », Genre, sexualité \& société, no 12 , http://journals. openedition.org/gss/3333.

Weber Florence, 2009, Manuel de l'ethnographe, Paris, Presses Universitaires de France, 334 p. 